章

SSCL-Preprint-409

May 1993

Distribution Category: 414

T. Garavaglia

\title{
Proton-Proton Scattering Contribution to Emittance Growth
}

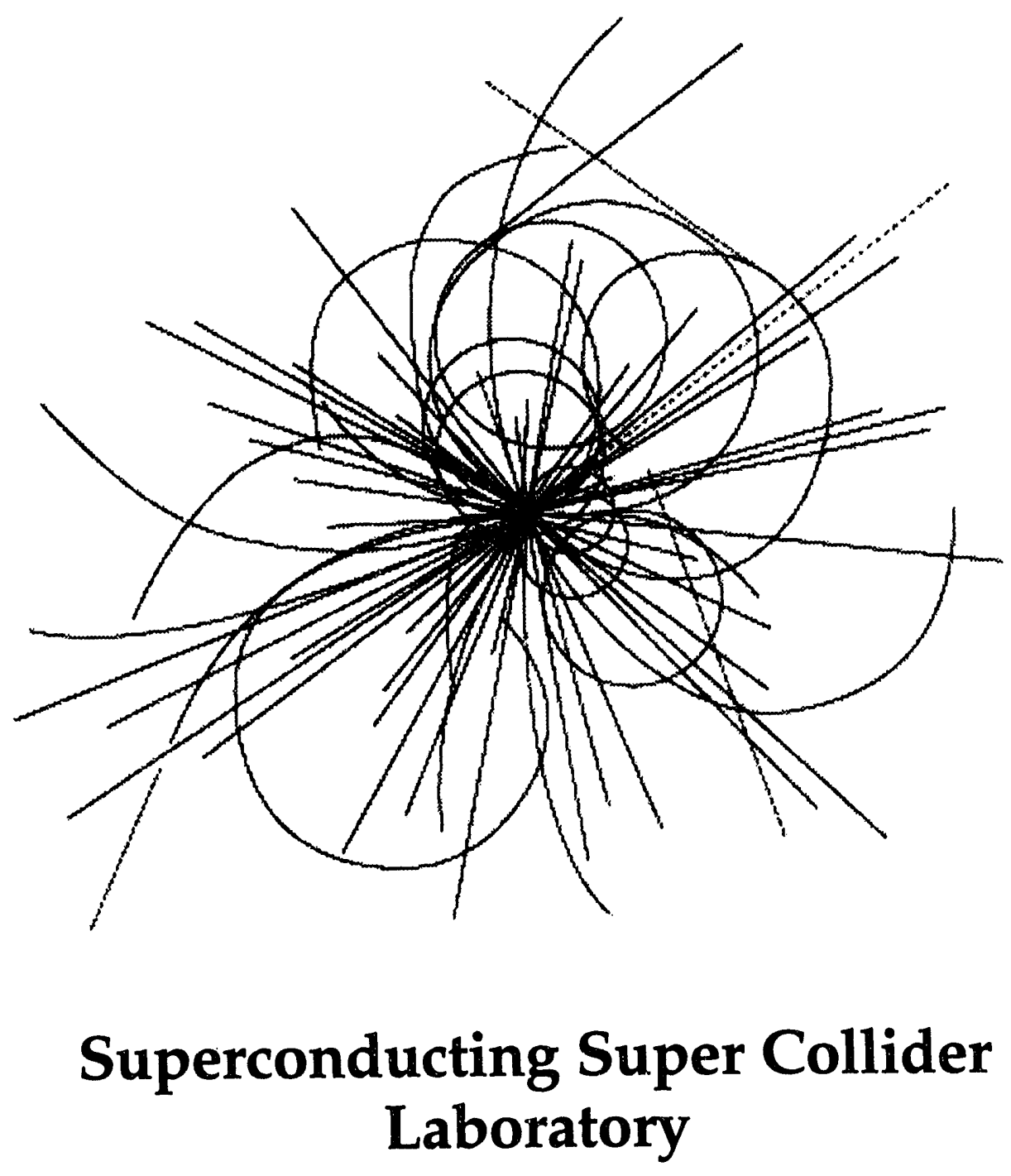

Juin 2053

$40 \%$ 


\section{Disclaimer Notice}

This ruport was prepared as an account of work sponsored by an agency of the United States Govemment. Neither the United States Government or any agency thereot, nor any of their omployees, makes any warranty, express or implied, or assumes any legal lisbility or responsibility for the accuracy, completeness, or usefuiness of any information, apparatus, product, or process disclosed, or represents that its use would not iniringe privately owned rights. Relerence herein to any specific commercial product, process, or service by trade name, trademark, manufacturer, or otherwise, does not necessarily constitute or imply its endorsement, recommendation, or favoring by the United States Government or any acency thereof. The views and opinions of authors expressed herein do nol necessarily state or reflect those of the United States Govemment or any agency thereot.

Superconducting Super Collider Laboratory is an equal opportunity employer. 


\title{
Proton-Proton Scattering Contribution to Emittance Growth*
}

\author{
T. Garavaglia \\ Superconducting Super Collider Laboratory ${ }^{\dagger}$ \\ 2550 Beckleymeade Avenue \\ Dallas, TX 75237
}

May 1993

\footnotetext{
* To be presented at the 1993 IEEE Particle Accelerator Conference on May 17-20, Washington, D.C.

$\dagger$ Operated by the Universities Research Association, Inc., for the U.S. Department of Energy under Contract No. DE-AC35-89ER40486. 


\title{
Proton-Proton Scattering Contribution to Emittance Growth
}

\author{
T. Garavaglia \\ Superconducting Super Collider Laboratory* \\ 2550 Beckleymeade Ave., Dallas, TX 75237
}

\begin{abstract}
Proton-proton scattering contributes to the emittance growth of the SSC. A formulation is given and used to estimate the mean scattering angle, which is used to determine the contribution to SSC emittance growth resulting from elastic $p p$ scattering. The method is based upon Lorentz invariants, and it permits the determination of the crosssection for scattering in the center of mass (c.m.) system, as well as scattering from a fixed target (f.t.). Also an example is given for the case of electromagnetic $p p$ scattering, which results from single virtual photon exchange.
\end{abstract}

\section{INTRODUCTION}

The contribution from $p p$ elastic scattering to transverse emittance growth is determined for the SSC. Elastically scattered protons with a small scattering angle will remain within the Collider proton beam. These scattered particles contribute to the growth of the beam's transverse emittance. Numerical results for emittance growth resulting from $p p$ scattering and other sources are given in [1]. In this paper, a summary is given of the analytical methods that are used to determine the mean scattering angle resulting from $p p$ elastic scattering. Lorentz invariants and cross-sections are defined in Appendix A.

\section{PROTON-PROTON ELASTIC SCATTERING}

The contribution to transverse emittance growth, for one degree of freedom, resulting from $p p$ elastic scattering is given by

$$
\left(\frac{d \epsilon_{x}}{d t}\right)=\left(\beta_{1 x}^{*} \mathcal{L}_{1}+\beta_{2 x}^{*} \mathcal{L}_{2}\right) \sigma_{e l}<\theta_{x}^{2}>/\left(M N_{B}\right)
$$

where $\beta_{i}^{*}$ and $\mathcal{L}_{i}$ are, respectively, the beta function and the luminosity at the $i^{\text {th }}$ interaction point. In this expression $N_{B}$ is the number of protons per bunch, $M$ is the number of bunches, $\sigma_{e l}$ is the $p p$ elastic scattering cross-section, and $\sqrt{\left\langle\theta_{x}^{2}\right\rangle}$ is the rms value of the $p p$ elastic scattering angle in the center of mass system, which is projected onto the transverse $x$-direction. A similar expression occurs for the transverse $y$-direction. The mean scattering angle can

-Operated by the Universities Research Association, Inc., for the U.S. Department of Energy under Contract No. DE-AC3589ER40486. be estimated from the differential elastic scattering crosssection

$$
\left(\frac{d \sigma_{e l}}{d \Omega}\right)_{c . m .}=\frac{S}{4 \pi}\left(\frac{d \sigma_{e l}}{d T}\right)_{c . m .},
$$

where $S$ and $T$ are Lorentz invariants. These invariants, defined in $(A 1)$, are $S \approx 2 E_{c . m .}$, and $T \approx-S \sin ^{2}(\theta / 2) \approx$ $-S\left(\theta_{x}^{2}+\theta_{y}^{2}\right) / 4$. The invariant differential cross-section for $p p$ elastic scattering is [2]

$$
\frac{d \sigma_{e l}}{d T} \approx \frac{\sigma_{T}^{2}}{16 \pi}\left(1+\rho^{2}\right) e^{b T}
$$

where $\sigma_{T}$ is the total $p p$ cross-section, $b$ is the slope parameter, and $\rho \approx 0$ is the ratio of the real part to the imaginary part of the scattering amplitude. Assuming that the slope parameter is a constant, one can integrate the differential cross-section to obtain $b \approx c_{T}^{\dot{\omega}} / 16 \pi \sigma_{\mathrm{cl}}$. With the approximation $\sigma_{e l} \approx(1 / 4) \sigma_{T}$, one finds $b \approx \sigma_{T} / 4 \pi$.

Using the approximations above, the differential crosssection in the c.m. system becomes

$$
\left(\frac{d \sigma_{e l}}{d \Omega}\right)_{c . m .} \approx \frac{S \sigma_{T}^{2}}{64 \pi^{2}} e^{\left(\frac{\theta_{T}^{2}}{2 \sigma_{\theta_{x}}^{2}}+\frac{\theta_{y}^{2}}{2 \sigma_{\theta_{x}}^{2}}\right)},
$$

where

$$
\sigma_{\theta_{x}}=\sqrt{<\theta_{x}^{2}>}=(b S / 2)^{-1 / 2} .
$$

This is the expression to be substituted into (2.1) to find $d \epsilon_{x} / d t$. For colliding proton beams with $\sqrt{S}=40 \mathrm{TeV}$ and $\sigma_{T} \approx 130 \mathrm{mb}$, one finds $b \approx 26.6 \mathrm{GeV}^{-2}$ and

$$
\sigma_{\theta_{x}}=\sqrt{\left.<\theta_{x}^{2}\right\rangle}=6.9 \mu \mathrm{rad}
$$

In the above, the value of the total $p p$ scattering crosssection is determined from

$$
\sigma_{T}=38.5+1.33 \ln ^{2}\left(\frac{\sqrt{S}}{10 \mathrm{GeV}}\right)
$$

which is obtained from cosmic ray data [3]. Theoretical models giving values for the total and elastic $p p$ crosssections can be found in [4].

Using the above techniques, one can estimate the rms scattering angle $\sqrt{\left\langle\tilde{\theta}^{2}\right\rangle}$ for the scattering of a proton in a $20 \mathrm{TeV}$ beam from a fixed proton. The scattering angle in the c.m. system for high energy $p p$ scattering is found from

$$
\cos (\theta) \approx \frac{2 T}{S}+1
$$


The scattering angle for a proton of energy $\omega=S / 2 m$ from a proton at rest is found from

$$
\cos (\tilde{\theta}) \approx 1-\frac{2 \sin ^{2}(\theta / 2)}{\left(S / m^{2}\right)}-\frac{4}{\left(S / m^{2}\right)^{2}}
$$

Using $\cos \tilde{\theta} \approx 1-(1 / 2) \sin ^{2} \tilde{\theta}$, the fixed target scattering angle is related to the c.m. scattering angle $\theta$ through

$$
\tilde{\theta} \approx \frac{\sqrt{2}}{(\omega / m)} \sqrt{\left(\frac{\theta^{2}(\omega / m)}{4}+1\right)} .
$$

For the scattering of a $20 \mathrm{TeV}$ proton from a proton at rest, the rms scattering angle in the c.m. system is found from (2.5), with $\sqrt{S}=193 \mathrm{GeV}, \sigma_{T}=50.2 \mathrm{mb}$ and $b \approx 10.3 \mathrm{GeV}^{-2}$, to be $\sqrt{\left.<\theta^{2}\right\rangle} \approx 2.3 \mathrm{mrad}$. The corresponding angle in the fixed target system is found to be $\sqrt{\left.<\tilde{\theta}^{2}\right\rangle} \approx 67 \mu \mathrm{rad}$.

\section{PROTON-PROTON ELECTROMAGNETIC SCATTERING}

In this example, $p p$ scattering is treated as an electromagnetic event, and the scattering of two fermions of initial four-momenta $a$ and $b$ to a final state of four-momenta $c$ and $d$ results from the exchange of a single virtual photon. The system of units $\hbar=c=m=1$ is used. Since both the initial and final states involve identical particles, these states must be antisymmetricai. The initial state $\mid I)$ and the final state $\mid F$ ) are represented as

$$
\mid I)=\frac{\mid a b)-(b a)}{\sqrt{2}}(F)=\frac{\mid c d)-|d c|}{\sqrt{2}} .
$$

The matrix element for this process is

$$
\begin{gathered}
(F|M| I)=[(c d|M| a b)-(c d|M| b a)+ \\
(d c|M| b a)-(d c|M| a b)] / 2 \\
=e^{2}\left[J^{\mu}(d, b) D_{\mu \nu}(a-c) J^{\nu}(c, a)-(d \leftrightarrow c)\right],
\end{gathered}
$$

where the fermion current is $J^{\mu}(c, a)=\bar{u}_{c} \gamma^{\mu} u_{a}$. The photon propagator is $D_{\mu \nu}(a-c)=4 \pi g_{\mu \nu} /\left((a-c)^{2}+i \epsilon\right)$. We use the fermion density matrix $\rho_{i j}(a)=u_{a i} \bar{u}_{a j}$, which has the property $\operatorname{Tr} \rho(a)=2$.

The invariant differential cross-section for this process is

$$
\frac{d \sigma_{e l}}{d T}=\frac{1}{j+\pi j(S, u, \dot{u})} \mathcal{M}(S, T),
$$

where $\mathcal{M}(S, T)=|(F|M| I)|^{2}$. For the scattering of unpolarized fermions when the polarization of the final state fermions is not observed, the initial state spin density matrices for $a$ and $b$ are of the form $\rho_{0}(a)=(\not+1) / 2$. For the final states $|c|$ and $(d)$, which include a summation over the final spin states, the density matrices are multiplied by two. The invariant differential cross-section for this case is now found to be

$$
\begin{aligned}
\frac{d \sigma_{e e}}{d T}(a b \rightarrow c d) & =\frac{\pi e^{4}}{64 S(S / 4-1)}[A(S, T, U)+A(S, U, T) \\
& -B(S, T, U)-B(S, U, T)]
\end{aligned}
$$

The invariant functions are

$$
\begin{aligned}
& A(S, T, U)=\frac{4}{T^{2}} T^{\mu \nu}(d, b) T_{\mu \nu}(c, a) \\
& B(S, T, U)=\frac{4}{T U} T_{\mu \nu}^{\mu \nu}(b, c, a, d)
\end{aligned}
$$

where

$$
\begin{gathered}
T^{\alpha \nu}(c, a)=\operatorname{Tr}\left[(k+1) \gamma^{\mu}(\phi+1) \gamma^{\nu}\right] \\
T^{\mu \nu \lambda \sigma}(b, c, a, d)=\operatorname{Tr}\left[\gamma^{\mu}(\phi+1) \gamma^{\nu}(k+1) \gamma^{\lambda}(\not+1) \gamma^{\sigma}(\phi+1)\right]
\end{gathered}
$$

Upon evaluation of the traces, the invariant functions become

$$
\begin{aligned}
& A(S, T, U)=\frac{32}{T^{2}}\left[S^{2}+U^{2}+8 T-8\right] \\
& \left.B(S, T, U)=-\frac{32}{T U}\left[S^{2}-8 S+12\right)\right] .
\end{aligned}
$$

In the high energy limit when $S$ becomes large, one finds

$$
\frac{d \sigma_{e l}}{d T}(a b \rightarrow c d) \approx \frac{2 \pi e^{4}}{T^{2}} .
$$

The rms value of the c.m. scattering angle associated with (3.11) can be found using (2.8) in the form

$$
<\cos \theta>\approx 1-<\theta^{2}>/ 2=2<T / S>+1
$$

where

$$
<T>=\int_{T_{\min }}^{T_{\max }} T\left(d \sigma_{e} / d T\right) d T / \sigma_{e},
$$

and $\sigma_{e}=\int\left(d \sigma_{e} / d T\right) d T$. The rms value of the scattering angle is written in terms of the projections on the transverse directions as $\sqrt{\left.<\theta^{2}\right\rangle}=\sqrt{\left.\left.\left(<\theta_{x}^{2}\right\rangle+<\theta_{y}^{2}\right\rangle\right) / 2}$. The integration limits are found from $T \approx \theta^{2} / 4$, where $\theta_{\max }$ is found from the condition that the proton is scattered from the beam, and where $\theta_{\min }$ is found from the uncertainty principal, $\Delta 2 \sigma_{\text {beam }} \Delta \theta \approx \hbar / p$. One finds $\sqrt{\left\langle\theta_{x}^{2}\right\rangle} \approx 1 . \mu \mathrm{rad}$.

\section{APPENDIX A: Kinematics and Cross-Sections}

In this appendix, the kinematical variables and crosssections used in the analysis are given.Particles characterized by four-momenta $a$ and $b$ interact elastically to yield particles characterized by four-momentum $c$ and $d$. For this process, energy-momentum conservation is represented as $a+b=c+d$, where a typical four-vector is represented as $a=\left(a^{0}, \mathbf{a}\right)$, such that $a^{2}=\left(a^{0}\right)^{2}-\mathbf{a} \cdot \mathbf{a}=m_{a}^{2}$. The 
interaction channels are defined according to the Lorentz invariants

$$
S=(a+b)^{2}, T=(a-c)^{2}, U=(a-d)^{2},
$$

which satisfy $(S+T+U)=a^{2}+b^{2}+c^{2}+d^{2}$.

In the c.m. system, one finds the invariant expressions for energy, momentum, and scattering angle

$$
\begin{gathered}
\mathcal{E}_{a}=\mathcal{E}(S, a, b)=\left(S+a^{2}-b^{2}\right) / 4(S / 4)^{1 / 2} \\
\mathcal{E}_{b}=\mathcal{E}(S, b, a), \mathcal{E}_{c}=\mathcal{E}(S, c, d), \mathcal{E}_{d}=\mathcal{E}(S, d, c), \\
|\mathbf{a}|=|\mathbf{b}|=\left[\frac{f(S, a, b)}{4 S}\right]^{1 / 2},|\mathbf{c}|=|\mathbf{d}|=\left[\frac{f(S, c, d)}{4 S}\right]^{1 / 2},
\end{gathered}
$$

and

$$
\cos \theta_{a c}=\left(\Gamma-a^{2}-c^{2}+2 \mathcal{E}_{a} \mathcal{E}_{b}\right), / 2|\mathbf{a}||\mathbf{c}|
$$

with

$$
f(S, a, b)=\left[S-\left(m_{a}+m_{b}\right)^{2}\right]\left[S-\left(m_{a}-m_{b}\right)^{2}\right]
$$

In the fixed target system, the corresponding relations are

$$
\begin{gathered}
\omega_{a}=\omega(S . a, b)=\left(S-a^{2}-b^{2}\right) / 2 m_{b}, \\
\omega_{b}=m_{b}, \omega_{c}=-\omega(U, c, b), \omega_{d}=-\omega(T, d, b), \\
|\mathbf{a}|=f^{1 / 2}(S, a, b) / 2 m_{b},|\mathbf{b}|=0, \\
|\mathbf{c}|=f^{1 / 2}(U, c, b) / 2 m_{b},|\mathbf{d}|=f^{1 / 2}(T, b, d) / 2 m_{b},
\end{gathered}
$$

and

$$
\begin{gathered}
\cos \tilde{\theta}_{a c}=\left[2 b^{2}\left(T-a^{2}-c^{2}\right)\right. \\
\left.-\left(S-a^{2}-b^{2}\right)\left(U-b^{2}-c^{2}\right)\right] /[f(S, a, b) f(U, c, b)]^{1 / 2}
\end{gathered}
$$

The differential cross-sections are found from the definition of the invariant total cross-section for the interaction of two particles initially in the states $|a\rangle$, and $|b\rangle$ and the subsequent production of an $n$-particle final state, where each particle is characterized by a momentum state $\left|p_{i}\right\rangle$.

This cross-section is defined as

$$
\begin{gathered}
\sigma(S, T)=\frac{1}{2 f^{1 / 2}(S, a, b)(2 \pi)^{3 n-4}} \times \int d p_{1} d p_{2} \ldots d p_{n} \\
\prod_{i=1}^{n} \delta\left(p_{1}^{2}-m_{1}^{2}\right) \theta\left(p_{i}\right) \times \delta\left(a+b-\sum_{i=1}^{n} p_{i}\right) \mathcal{M}(S, T, U),
\end{gathered}
$$

with $\theta(p)=\left[\left(p^{0} / \omega\right)+1\right] / 2, \omega=\left(|\mathbf{p}|^{2}+m^{2}\right)^{1 / 2}$, and

$$
\mathcal{M}(S, T, U)=\left.|<f| \mathcal{M}|a, b\rangle\right|^{2},
$$

where $<|M| a, b>$ is the transition amplitude from the initial to the final state. In (A9), one uses an invariant definition of the flux, which is represented as the magnitude of the relative velocity $\left|\mathbf{v}_{a}-\mathbf{v}_{b}\right|$ in the c.m. system. The flux becomes $F=f^{1 / 2}(s, a, b) / 2 \mathcal{E}_{a} \mathcal{E}_{b}$.

Particular differential cross-sections may now be obtained from (A9). Of special interest is the differential cross-section defined formally as

$$
\frac{d \sigma}{d T}=\sigma(S, T) \delta\left[T-(a-c)^{2}\right]
$$

For scattering into the solid angle $d \Omega_{a c}$, one finds for elastic scattering in the c.m. system

$$
\frac{d \sigma}{d \Omega_{c . m .}}=\frac{1}{4 \pi S} f^{1 / 2}(S, a, b) f^{1 / 2}(S, c, d) \frac{d \sigma}{d T} .
$$

The corresponding differential cross-section in the f.t. system may be found from (A8) and (A10) to be

$$
\frac{d \sigma}{d \Omega_{f . t}}=\frac{-2 f^{1 / 2}(S, a, b) f^{3 / 2}(U, c, b)}{\pi g(S, T, 1, m, 1, m)} \frac{d \sigma}{d T}
$$

For the elastic scattering of a particle of unit mass with a particle of mass $m$, one finds the expression

$$
\begin{gathered}
g(S, t, 1, m, 1, m)=128 m^{2}\left[(S / 4)^{2}+S T / 16-(S / 4)\left(m^{2}+1\right) / 2\right. \\
\left.-(T / 4)\left(m^{2}-1\right) / 4+\left(m^{2}-1\right)^{2} / 16\right] .
\end{gathered}
$$

The integration indicated in (A9) and (A10), when there is a two-particle final state characterized by four-momenta $c$ and $d$, is performed in the c.m. system using the mornentum-space measure

$$
d c d d=|\mathrm{c}|^{2} d|\mathrm{c}| d \Omega_{c} \frac{d c^{2}}{2 c^{0}} d \mathrm{~d} \frac{d d^{2}}{2 d^{0}}
$$

to find

$$
\frac{d \sigma}{d T}=\frac{1}{16 \pi f(S, a, b)} \mathcal{M}(S, T) .
$$

\section{REFERENCES}

[1] W. Chou, S. Dutt, T. Garavaglia, and S. K. Kauffmann, these proceedings.

[2] K. Goulianos, "Diffractive and rising cross-sections," Comments on Nucl. Part. Phys.17, No. 4, pp. 177-193 (1987).

[3] M. Honda et.al., Phys. Rev. Lett. 70, 525 (1993).

[4] P.V. Landshoff, "Soft hadron physics," Joint International Lepton-Photon \& Europhysics Conference on High Energy Physics, Vol. 2, pp. 365-373 (World Scientific, 1991). 

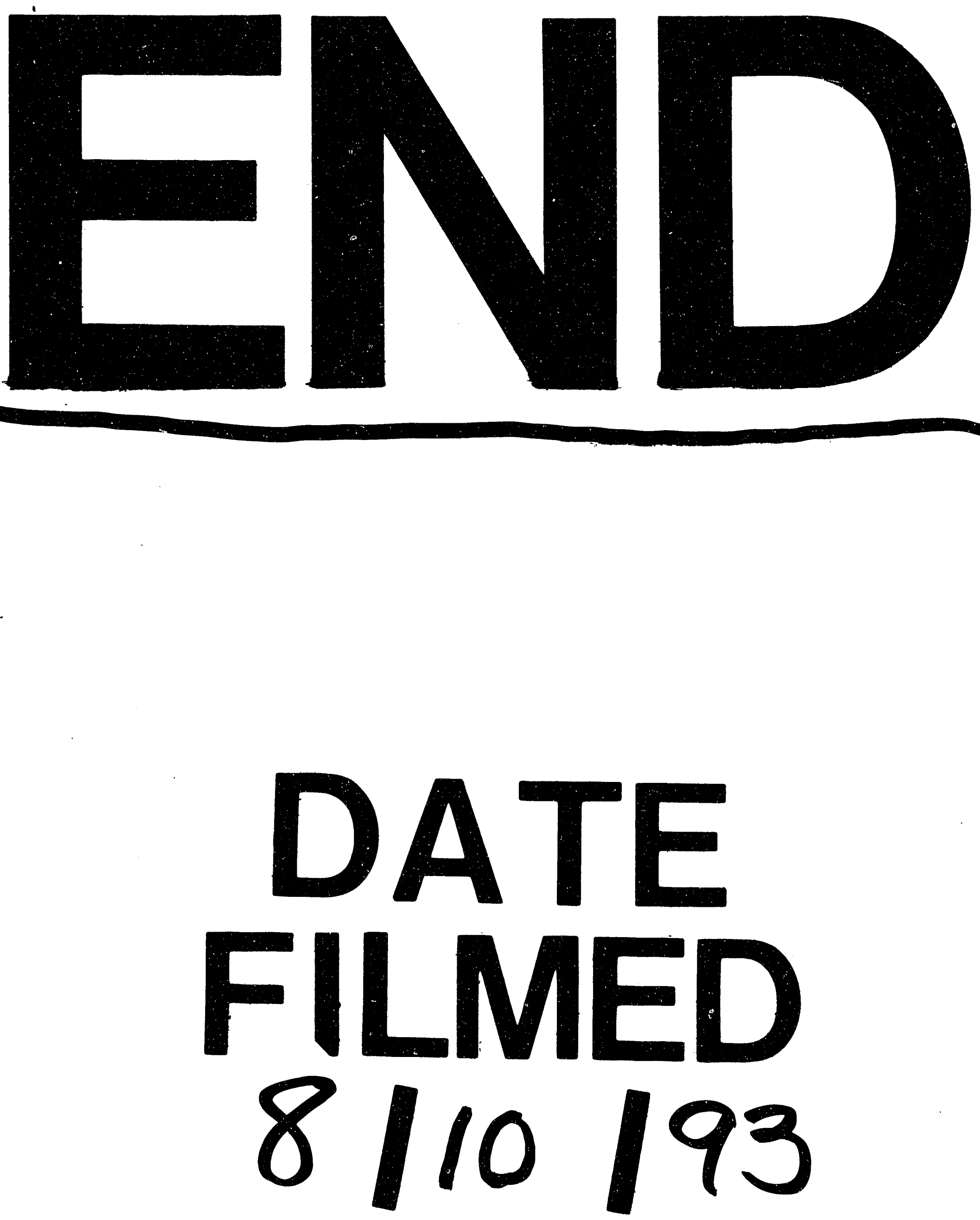
